\title{
Study of Distributed Resource Allocation in Cellular Systems
}

\author{
S. Anand, A. Sridharan, K. N. Sivarajan. \\ Department of ECE, Indian Institute of Science, \\ Bangalore- 560012 , India. \\ Ph: 91-80-309-2658, Fax: 91-80-360-0991 \\ anand@cellular.ece.iisc.ernet.in
}

\begin{abstract}
Dynamic Channel allocation in Cellular Networks have become very popular off late due to the flexibility obtained by using any of the available channels in any cell as long as the Interference level is below a given threshold. Distributed Resource Allocation is popular in modern day cellular systems due to the increase in micro-cells and picocells. In our problem we study Cellular networks under Distributed Dynamic Channel Allocation and propose a Two Dimensional Markov Chain model to analyze the performance of the system in terms of Blocking probabilities. In our analysis, we consider all the mobiles and base stations to be perfectly power controlled, i.e. each Mobile (Base station) transmits on any channel. such that the signal strength received at its corresponding Base station (Mobile) is unity irrespective of the actual position of the Mobile in the cell. We perform this analysis for a linear highway system and a two dimensional cellular system modelling the shape of the cells to be perfect circles. We look at low mobility systems in our problem. In practice, Digital European Cordless Telephony (DECT) is a standard to which our analysis is applicable. Our Analysis is also applicable for a GSM like system along a busy street and with low mobility. Keywords : Cellular Networks, Highway Systems, Dynamic Channel Allocation (DCA). Distributed, Power Control
\end{abstract}

\section{Introduction}

Distributed Resource Allocation Schemes are becoming popular in Cellular Systems keeping in view that the cellular systems of the future are going to have more of micro cells and pico cells. Cellular Systems with Dynamic Channel Allocation (DCA) have been studied for blocking probability performances with Erlang B approximations in each cell by Cimini et.al[1]. In that, they had analyzed DCA systems with Centralized and Distributed Channel Allocation and had shown that Distributed Channel Allocation im- proves the system performance by one order of magnitude. We had devised an anlytical tool in [2] to compare centralized and distributed schemes for linear highway systems without Rayleigh Fading and Shadowing to show that distributed channel allocation schemes indeed perform better than the centralized schemes. We now analyze linear highway systems and circular cellular systems with powerloss due to distance attenuation and log normal shadowing. We propose a two dimensional Markov chain for each cell with $\frac{n(n+1)}{2}$ states and solve this chain $N$ times for $N$ cells and once if all cells have uniform traffic. If we consider a Cellular Network along a busy street, then the base stations of this network are collinear and such a system is called a Linear Highway System. By extending the geometry to consider positions of individual mobiles on a two dimensional circular plane we have a Circular Cellular System. We compute the blocking probability in such systems.

\section{Organization}

The rest of the paper is organized as follows. In section 3 , we define the actual objective of our problem and the parameters to be computed. Section 4 gives the mathematical model for our problem and section 5 deals with the analysis of our model to obtain expressions and values for blocking and outage probabilities. In section 6 , we compare the results obtained by our analytical model against computer simulations and finally, in section 7 , we give our conclusions and scope for further work in this problem.

\section{Problem Definition}

A typical Highway Cellular System is as shown in figure 1. $\alpha_{1}, \alpha_{2}, \ldots, \alpha_{N}$ denote the positions of the base-stations in the highway of length $d$. A typical Circular Cellular System is shown in figure 2. The problem is to device an analytical model for computing the blocking probability in the system Distributed Dynamic Channel Allocation. We 


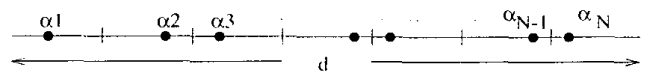

Figure 1. A Typical Highway Cellular System.

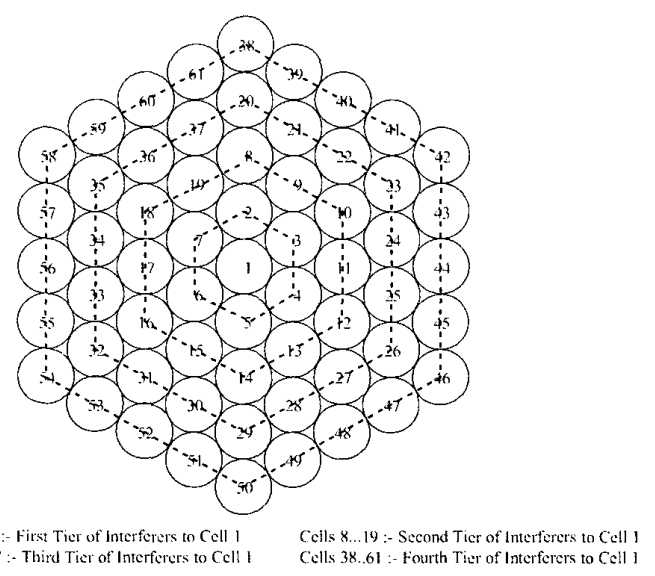

Figure 2. A Typical 61 Cells Circular Cellular System.

are given the length of the highway $d$ and the number of cells, $N$ for a Linear Highway System and the Cell Radius $R$ for the Circular Cellular System. The Channel Allocation strategy is as follows. Each base station measures the power it receives from mobiles of all other cells at all the channels. The mobiles also measure the power they receive from all the base-stations at all the channels. The base-stations (mobiles) are power controlled as in [2]. Hence the parameter the base stations and mobiles really measure is the ratio between the Interference and Signal (denoted by $\frac{1}{S}$ ). The $\frac{1}{S}$ at every channel measured by the mobiles and the base-station at every channel is compared with a threshold $\epsilon$ and a new call is alloted one of the channels that has $\frac{I}{S}<\epsilon$. We call those channels which have $\frac{I}{S}<\epsilon$ as available channels or feasible channels. We allot any one of the available channels at random. The difference between centralized and distributed schemes and the different possible channel allocation schemes are mentioned in [2].

* - Here interference level means the $\frac{T}{S}$ level. Henceforth, throughout the paper, the terms "interference lever" and " $\frac{I}{S}$ level" will be used interchangeably unless specfied otherwise.

\section{Mathematical Model}

\section{Given parameters}

The parameters that are known are, the number of cells $N$ and the number of channels $n$. For the highway system we know the length of the highway $d$. Similarly, for the Circular System, we know the cell radius $R$.

Assumptions made in our analysis.

- All cells are assumed to be of equal length $\frac{d}{N}$ in the highway system and of equal radius $R$ in the circular system, with the base-stations situated at the centers of each cell.

- The positions of a call in any cell is uniformly distributed over the length of the cell for the highway system and over the area of the cell for the circular system.

- The call arrival process in any cell is a Poisson process with rate $\lambda$.

- The call holding time in any cell is Exponentially distributed with mean $\frac{1}{\mu}$.

- The signal undergoes Distance Attenuation and Log Normal Fading.

The RF signal that propagates in the air interface undergoes a short term Rayleigh fading $R_{f}$, long term Log normal fading $G$ and attenuation due to the distance between the mobile and the base-station. Hence if unit power transmitted by the mobile (base-station) which is at a distance $d_{0}$ from the base-station (mobile) the power received by the base-station (mobile) $P_{r}$ is given by $P_{r}=\phi R_{f}^{2} G^{2} d_{0}^{-4}$. where $\phi$ is a proportionality constant. We average out the Rayleigh Fading Compoenent in our analysis making the average value of $R_{f}^{2}$ unity.

\section{Analysis}

In our analysis, since we are looking at the ratio of the interference power to signal power, we need to only consider the ratio of the distances of the mobiles from the basestations and vice-versa.

\subsection{Linear Highway System}

Let $x_{i}$ be the random variable that denotes the position of the mobile in cell $i$. Hence, if only the mobiles in the immediate neighboring cells cause signi ficant interference. For any two cells $M$ and $M+1$ that have calls going on at positions $x_{M}$ and $x_{M+1}$, the $\frac{I}{S}$ at the mobiles due to each other and at the base-stations $\alpha_{M}, \alpha_{M+1}$, are given by

$$
\begin{gathered}
\gamma_{B S_{M}}=\left(\frac{x_{M+1}-\alpha_{M+1}}{x_{M+1}-\alpha_{M}}\right)^{4} 10^{\frac{-t^{\prime} M+1 M+1}{10}} 10^{\frac{-\psi_{M+1 M}}{10}} \\
\gamma_{B S_{M+1}}=\left(\frac{x_{M}-\alpha_{M}}{x_{M}-\alpha_{M+1}}\right)^{4} 10^{\frac{-\psi_{M M}}{10}} 10^{\frac{-\psi_{M M+1}}{10}}
\end{gathered}
$$

Equations $I$ and 2 are obtained by taking power control into consideration. $\psi_{i j}$ denotes the fading component at base 
station in cell $j$ due to a mobile in cell $i . \psi$ is a Normally distributed random variable with zero. mean and variance $\sigma^{2}$. The probability that the call at $x_{M}$ can simultaneously not use the same channel as that of the call at $x_{M+1}$ is given by

$$
\int_{x_{M+1}=\frac{M d}{N}}^{x_{M+1}=\frac{(M+1) d}{N}}\left(\frac{N}{d}\right) Q\left[\frac{\epsilon_{0}-\mu}{\sigma}\right] d x_{M+1}
$$

where, $\mu=\gamma_{B S_{M}}$ as in equation 1 , and $\epsilon_{0}=\ln (\epsilon)$ and $Q(x)=\int_{x}^{\infty} \frac{1}{\sqrt{2 \pi}} e^{\frac{-u^{2}}{2}} d u$. However if the set of interfering cells for cell $i$ is given by $S_{i}$ and we have $\Delta$ number of interferers to cell $i$, The probability that the $\frac{I}{S}>\epsilon$ at the base station in cell $i$ is given by

$$
p_{\text {thresh }}^{(i)}(\Delta, \epsilon)=\operatorname{Pr}\left\{I_{i}>\epsilon\right\}
$$

where $I_{i}$ is the $\frac{I}{S}$ at the base-station of cell $i$ given by

$$
I_{i}=\sum_{\substack{j \in S_{i} \\\left|S_{i}\right|=\Delta}}\left(\frac{x_{j}-\alpha_{j}}{x_{j}-\alpha_{i}}\right)^{4} 10^{\frac{-\psi_{j j}}{10}} 10^{\frac{-\psi_{j i}}{10}}
$$

In our analysis we model that significant interference is caused only by the immediate neighbouring cells. Hence $1 \leq \Delta \leq 2$ and for the interference caused at the base station of cell $M$ due to calls going on in both cells $M-1$ and $M+1$, we compute $p_{\text {thresh }}$ by the Fenton Wilkinson's method[3] to be

$$
p_{\text {thresh }}(\Delta, \epsilon)=\iint F^{(c)(M)}(\Delta, \epsilon) d x_{M+1} d x_{M-1}
$$

where $F^{(c)(M)}(\Delta, \epsilon)$ is the complementary cdf of the $\frac{I}{S}$ at the base station of cell $M$ given by,

$$
\left(\frac{N}{d}\right)^{\Delta} Q\left[\frac{\epsilon_{0}-\mu_{\Delta}}{\sigma_{\Delta}}\right]
$$

where $\sigma_{\Delta}$ is given by

$$
\sigma_{\Delta}^{2}=\frac{1}{a^{2}} \ln \left[1+\frac{\left(e^{a^{2} \sigma^{2}}-1\right) \sum_{i=1}^{\Delta} e^{-2 a \mu_{i}}}{\left(\sum_{i=1}^{\Delta} e^{-a \mu_{i}}\right)^{2}}\right]
$$

and $\mu_{\Delta}$ by

$$
\mu_{\Delta}=\frac{a}{2}\left[\sigma_{\Delta}^{2}-\sigma^{2}\right]-\frac{1}{a} \ln \left[\sum_{i=1}^{\Delta} e^{-a \mu_{i}}\right]
$$

where $a=\frac{\ln (10)}{10}$ and $\mu_{2}=\gamma_{B S_{M}}$ as in equation 1 and $\mu_{1}$ is same as $\mu_{2}$ except that $M+1$ is replaced by $M-1$.

\subsection{Circular Cellular System}

The analysis for computing $p_{\text {thresh }}^{(i)}(\Delta, \epsilon)$ for any cell $i$ in a circular cellular system is simlar to that of the Linear Highway System except that the expression for $\frac{I}{S}$ at cell $i$, $I_{i}$ is given by

$$
\begin{array}{r}
I_{i}=\sum_{\substack{j \in S_{i} \\
\left|S_{i}\right|=\Delta}} D^{2}(i, j) 10^{\frac{-\psi_{j j}}{10}} 10^{\frac{-\psi_{j i}}{10}} \\
D(i, j)=\left(\frac{r_{j}^{2}+r_{\alpha_{j}}^{2}-2 r_{j} r_{\alpha_{j}} \cos \left(\theta_{j}-\theta_{\alpha_{j}}\right)}{r_{j}^{2}+r_{\alpha_{i}}^{2}-2 r_{j} r_{\alpha_{i}} \cos \left(\theta_{j}-\theta_{\alpha_{i}}\right)}\right)
\end{array}
$$

where $r_{j}$ is the $r$-coordinate of the position of a mobile in cell $j$ and $\theta_{j}$ is the $\theta$-coordinate. $r_{\alpha_{j}}, \theta_{\alpha_{j}}$ are the $r$ and $\theta$ coordinates respectively of the base station of cell $j \cdot p_{\text {thresh }}$ is given by,

$$
\begin{aligned}
& p_{\text {thresh }}(\Delta, \epsilon)=\int \ldots \int F^{(c)(M)}(\Delta, \epsilon) r_{1} \ldots r_{\Delta} \\
& d r_{1} \ldots r_{\Delta} d \theta_{1} \ldots \theta_{\Delta}
\end{aligned}
$$

with $F^{(c)(M)}$ as given in equation 6 with $\frac{N}{d}$ replaced by $\frac{1}{\pi R^{2}}$. The expressions for $\mu_{\Delta}$ and $\sigma_{\Delta}^{2}$ are similar to that of the Linear Highway system case, with $\mu_{j}$ given by $\operatorname{10log}_{10}\left[D^{2}(i, j)\right]$.

\subsection{Computing Blocking Probability}

For the distributed channel Channel Allocation scheme, we model the state of each cell as a 2-dimensional CTMC. Each state $(m, k)$ in the CTMC denotes $m$ channels in use in a cell and $k$ channels being unusable due to violation of the interference constraint. The CTMC with its transition rates is shown in figure 3 . The CTMC in figure 3 cannot be solved analytically. Hence we modify it to take the form as shown in figure 4 . This is because, if the $\frac{I}{S}$ on a particular channel is close to threshold, then, the probability of another call getting this same channel in the neighboring cell is low. For the CTMC shown in figure 4, the expression for the probability of occupying a given state $\pi(m, k)$ at steady state is given by [4]

$$
\pi(m . k)=\frac{1}{G}\left(\frac{\lambda}{\mu}\right)^{m} \frac{1}{m !}\left(\begin{array}{l}
n \\
k
\end{array}\right) \zeta^{k}
$$

where $G$ is a normalization term given by

$$
G=\sum_{m+k \leq n}\left(\frac{\lambda}{\mu}\right)^{m} \frac{1}{m !}\left(\begin{array}{l}
n \\
k
\end{array}\right) \zeta^{k}
$$



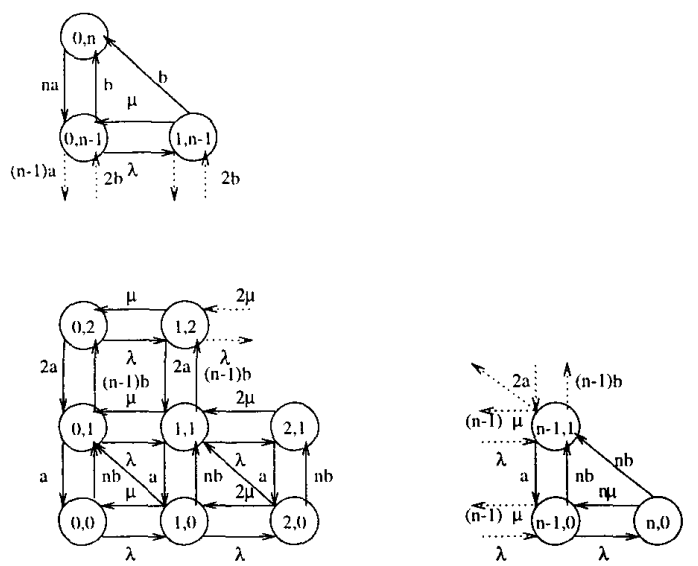

Figure 3. State Transition Diagram for Channel Occupancy in a Cell for the Distributed Channel Allocation Scheme.
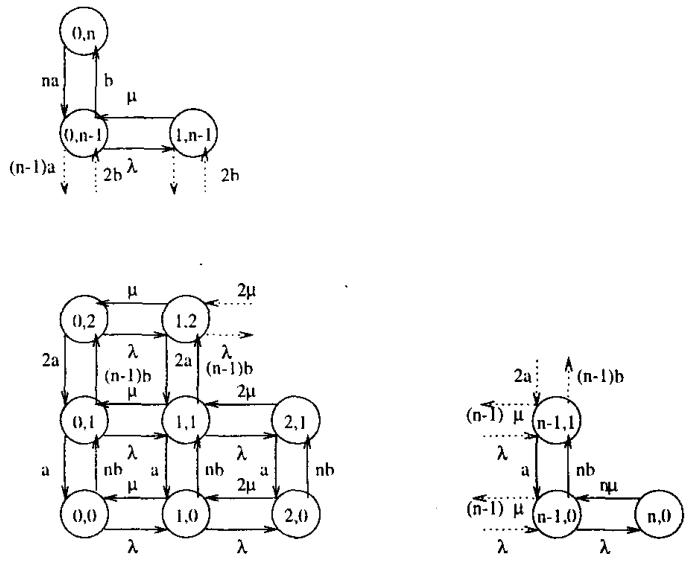

Figure 4. Modified State Transition Diagram for Channel Occupancy in a Cell for the Distributed Channel Allocation Scheme.

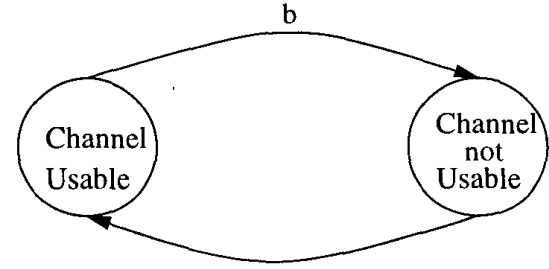

a

Figure 5. СTMC model for channel interference level crossing the threshold $\epsilon$.

For computing $\zeta$, we model the process where a channel is usable or not usable as a CTMC as shown in figure 5. We define $\xi$ as the probability that a channel $k$ is usable in cell $i$. This is identical for all channels in all cells due to the frequency non-selective propagation characteristics and random channel allocation. If only the two immediate neighbors cause significant interference, and the probability of using a channel in a cell be $p$, then, $\xi$ is given by,

$$
1-\xi=\sum_{\delta=1}^{\Delta}\left(\begin{array}{l}
\Delta \\
\delta
\end{array}\right) p^{\delta}(1-p)^{(\Delta-\delta)} p_{\text {thresh }}(\delta, \epsilon)
$$

We then compute $\zeta$ as,

$$
\zeta=\frac{b}{a}=\left(\frac{1-\xi}{\xi}\right)
$$

The probability that a channel is being used in a cell, $p$ is given by

$$
p=\sum_{m+k \leq n}\left(\frac{m}{n}\right) \pi(m, k)
$$

We compute the blocking probability $P_{B}$ as

$$
P_{B}=\sum_{m+k=n} \pi(m, k)
$$

In equation $9, \Delta=2$ for the linear system and 6 for the circular system. Note that the CTMC structure is same for both the Linear Highway System and the Circular System, and what varies is the computation of $p_{\text {thresh. }}$. The maximum value of $\Delta$ is 2 for the Linear System and 6 for the Circular System as we are considering interference only from the immediate neighbors to a cell. This is also the expression for Intra Cell Handoff Blocking Probability, since we do not reserve any channels for handoffs. 


\section{Results}

We have analyzed the distributed channel allocation scheme for the linear highway system and circular cellular system and compared them with simulations too. We have made our computations for the following values. $\lambda=2,3$ $\mathrm{s}^{-1}, \mu=0.01 \mathrm{~s}^{-1}, d=1$ for the linear system and and $R=1$ for the circular system. $N=12$ and 61 for the linear and circular systems respectively. We have obtained results for the 12 cell highway systems and 61 cell circular system. It is to be noted from equations 8,11 and 9 , that we need to run a fixed point iteration to compute $\pi(m, k)$. The computations were done with base station alone making measurements. We have computed blocking and handoff blocking probabilities for a load $\rho$ of 200 and 300 Erlangs. as shown in figures $6,7,8$ and 9

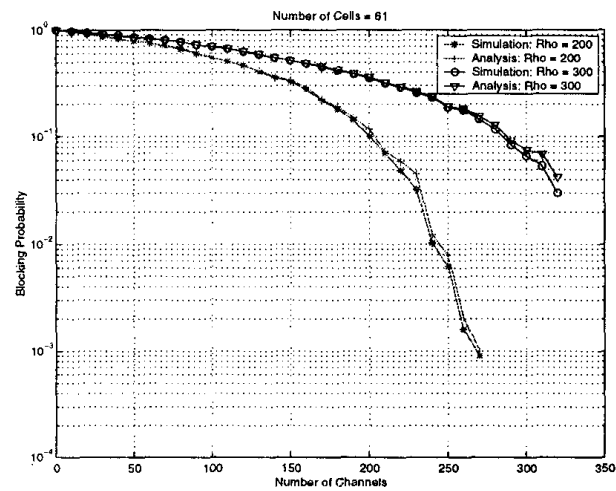

Figure 6. Comparison of Analysis and Simulation for Blocking Probability in a 12 Cell Linear Highway System

Figures 6 and 8 show that our analysis matches simulation results well hence validating our CTMC model for the linear and circular systems with shadowing. Our method of analysis has already been validated for systems without shadowing and compared with other methods[2]. It was mentioned at the end of section 5.3 that since we do not reserve any channels for handed off calls, the blocking probability we obtain is also the same as the handoff blocking probability. This claim is validated by comparision with simulations in figures 7 and 9 . We also note that our model in sections 5.1 and 5.2 that only immediate neighbours cause significant interference is validated by the plots in figures $6 \cdots 9$. Figures $6 \cdots 9$ also suggest that both for the Linear Highway System and the Circular Cellular System with one significant interfering neighbor, our analysis over estimates the blocking probability computed by simulations. From the expressions for $p_{\text {thresh }}$ for the lin-

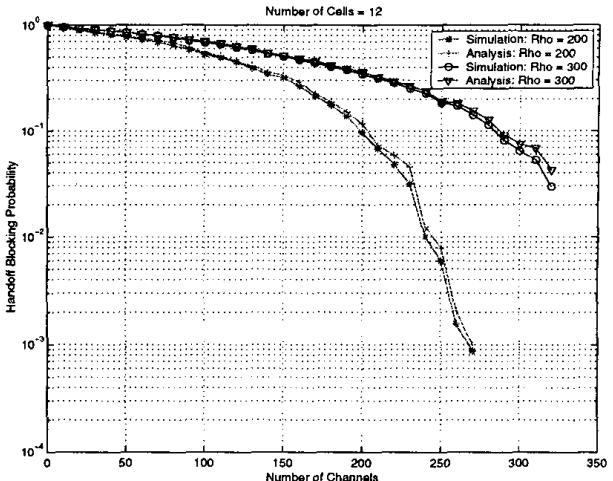

Figure 7. Comparison of Analysis and Simulation for Handoff Blocking Probability in a 12 Cell Linear Highway System.

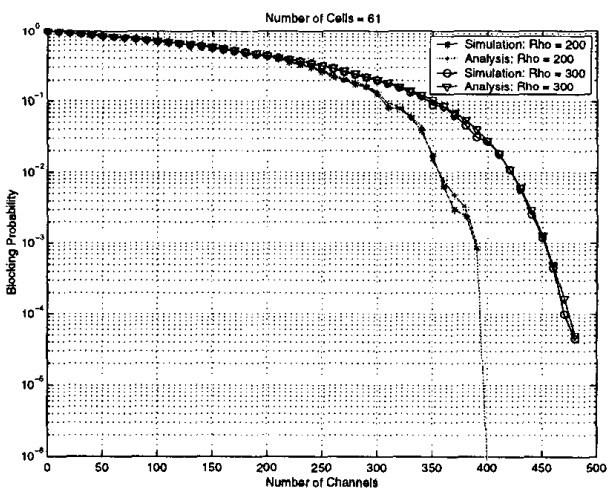

Figure 8. Comparison of Analysis and Simulation for Blocking Probability in a 61 Cell Circular Cellular System. 


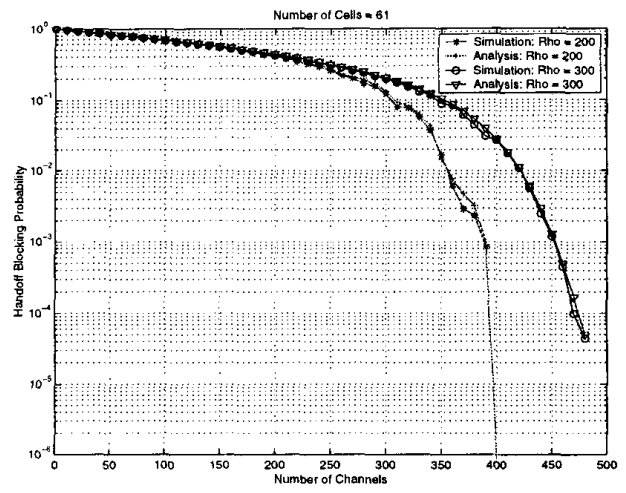

Figure 9. Comparison of Analysis and Simulation for Handoff Blocking Probability in a 61 Cell Circular Cellular System.

ear and circular systems, we note that $p_{\text {thresh }}$ and hence the blocking probability is a function of $N$ and $d$ for the linear system. However, for a fixed $\frac{d}{N}$ i.e., for a fixed cell length, the system performance remains same for any number of cells. This is not obvious from the expression for $p_{\text {thresh }}$ for the circular systems, but there too, if we constrain the area of the entire region i bounded by all the cells to a fixed constant $A$, then, a similar argument like that for the linear highway system holds. Thus, the analysis could be done for fewer cells for the same cell length or cell radius.

\section{Conclusion and Future Work}

We have discussed the analysis of a Linear Highway System and Circular Cellular Systems. We have devised a tool for computing the blocking probability for these two systems with distributed allocation scheme. We propose to optimize the number of cells for a specified blocking performance since we have discussed that the blocking probability is a function of the cell dimensions. We propose to extend our analysis to devise Admission Control Strategies in CDMA and $3 \mathrm{G}$ systems.

\section{References}

[1] Leonard Cimini, Gerard Foschini, Chih-Lin I, Zoran Miljanic, "Call Blocking Performance of Distributed Algorithms for Dynamic Channel Allocation in Micro Cells", IEEE Transactions on Communications, Vol.42, No.8, 1994

[2] S. Anand, A. Sridharan, K. N. Sivarajan, "Blocking Probability Analysis of Cellular Systems under
Dynamic Channel Allocation." IEEE, International Conference on Wireless Personal Communications (ICPWC) 2000, December 2000.

[3] L. F. Fenton, "The Sum of Log-Normal Probability Distributions in Scatter Transmission Systems." IRE Transactions on Communication Systems, CS-8, March 1960.

[4] Keith W. Ross, "Mulitservice Loss Models in Broadband Telecommunication Networks." SpringerVerlag. 